\title{
First-Order Magnetic Phase Transition of Mobile Electrons in Monolayer $\mathrm{MoS}_{2}$
}

\author{
Jonas G. Roch $\odot,{ }^{1, *}$ Dmitry Miserev $\odot,{ }^{1}$ Guillaume Froehlicher, ${ }^{1}$ Nadine Leisgang $\odot,{ }^{1}$ Lukas Sponfeldner, ${ }^{1}$ \\ Kenji Watanabe $\odot,{ }^{2}$ Takashi Taniguchi, ${ }^{2}$ Jelena Klinovaja, ${ }^{1}$ Daniel Loss, ${ }^{1}$ and Richard J. Warburton ${ }^{1}$ \\ ${ }^{1}$ Department of Physics, University of Basel, Klingelbergstrasse 82, CH-4056 Basel, Switzerland \\ ${ }^{2}$ National Institute for Material Science, 1-1 Namiki, Tsukuba 305-0044, Japan
}

(Received 14 November 2019; accepted 3 April 2020; published 8 May 2020)

\begin{abstract}
Evidence is presented for a first-order magnetic phase transition in a gated two-dimensional semiconductor, monolayer- $\mathrm{MoS}_{2}$. The phase boundary separates a ferromagnetic phase at low electron density and a paramagnetic phase at high electron density. Abrupt changes in the optical response signal an abrupt change in the magnetism. The magnetic order is thereby controlled via the voltage applied to the gate electrode of the device. Accompanying the change in magnetism is a large change in the electron effective mass.
\end{abstract}

DOI: 10.1103/PhysRevLett.124.187602

Mobile electrons in a semiconductor can lower their energy by aligning their spins, a consequence of the Pauli principle. A ferromagnetic phase in which all electron spins point in the same direction was proposed originally by Bloch [1]. Experimental verification of this prediction on two-dimensional (2D) electron gases in conventional semiconductors was elusive [2,3] on account of disorder [4,5]. Recently however, ferromagnetic ordering of 2D electrons was reported in monolayer $\mathrm{MoS}_{2}$ [6] and twisted-bilayer graphene [7]. A phase transition can be expected between a ferromagnetic state at low density and a paramagnetic state at high density. The behavior on crossing the phase boundary is crucial: does the magnetization turn on abruptly or does the magnetization increase gradually?

In three-dimensional (3D) ferromagnets such as iron, the magnetization grows gradually from zero on crossing the phase boundary. This is classified as a "second-order" phase transition. An abrupt change in the magnetization, a "first-order" phase transition, is more striking and is observed in more exotic metals, so-called "quantum ferromagnets" [8]. This is potentially much more useful in spintronics: a small change in a control parameter from one side of the phase boundary to the other results in a massive change in the magnetization. In metallic systems, the control parameter is typically the temperature or pressure, neither convenient for fast and efficient switching from one phase to the other. This restriction is lifted in $2 \mathrm{D}$ semiconductors for which the electron density can be controlled over a wide range simply via a voltage applied to a gate

Published by the American Physical Society under the terms of the Creative Commons Attribution 4.0 International license. Further distribution of this work must maintain attribution to the author(s) and the published article's title, journal citation, and DOI. electrode. However, magnetism of mobile electrons in two dimensions is different to that in three dimensions. On the one hand, mean-field theories, such as those of Bloch [1] and Stoner [9], are provably inadequate in two dimensions $[10,11]$. On the other hand, corrections to Fermi liquid theory, effects which can result in a first-order phase transition, are predicted to be much more pronounced in two dimensions compared to three dimensions [8,12-14]. Experimentally, magnetic phase transitions of mobile electrons in 2D semiconductors are unexplored.

We focus on monolayer $\mathrm{MoS}_{2}$, a 2D semiconductor in the transition-metal dichalcogenide (TMDC) family. We present evidence for a first-order phase transition between a paramagnetic phase at high electron density and a ferromagnetic phase at low electron density. The magnetism is thereby controlled electrically simply via the voltage applied to a gate electrode. Accompanying this abrupt change in magnetization is an abrupt change in the electron effective mass, from a relatively small value in the paramagnetic phase to a large value in the ferromagnetic phase.

The conduction band structure of $\mathrm{MoS}_{2}$ exhibits minima at the edges of the Brillouin zone, at the $K$ and $K^{\prime}$ points [15-17]. The spin- $\uparrow$ and spin- $\downarrow$ states are split by a small spin-orbit interaction, $\Delta_{\mathrm{CB}}$ [Fig. 1(a)]. Calculations predict $\Delta_{\mathrm{CB}}=3 \mathrm{meV}$ [17]; experiments suggest $\Delta_{\mathrm{CB}}=0.8 \mathrm{meV}$ [18]. The bare electron mass is relatively large, $0.44 m_{o}$ according to theory [17]; the dielectric constant relatively small, such that the Bohr radius is just $\sim 0.5 \mathrm{~nm}$, only slightly larger than the lattice constant. Quantum effects in gated $\mathrm{MoS}_{2}$ have recently been reported: conductance quantization [18], Shubnikov-de Haas oscillations [19], and ferromagnetic spin ordering [6].

Here, a monolayer of $\mathrm{MoS}_{2}$ is embedded between two hBN layers. Electrons are injected into the $\mathrm{MoS}_{2}$ by applying a voltage to a metallic contact [Fig. 1(b)]. The ground state of the mobile electrons is probed optically 


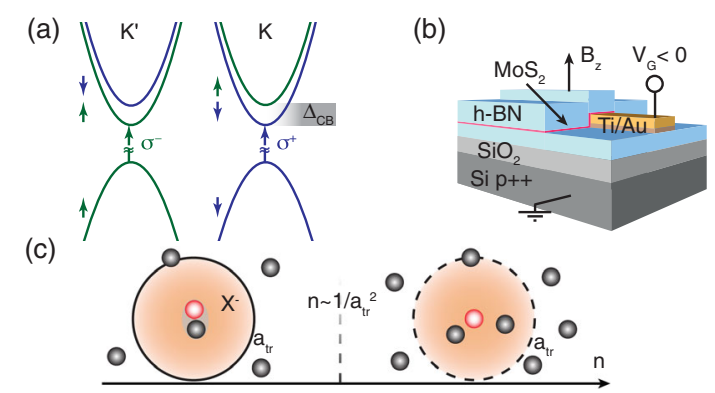

FIG. 1. (a) Band structure and allowed optical transitions of monolayer $\mathrm{MoS}_{2}$. (b) A van der Waals heterostructure consisting of monolayer $\mathrm{MoS}_{2}$ embedded in h-BN. (c) Schematic of a trion in a two-dimensional Fermi sea. The black circles denote conduction-band electrons; the red circle a valence-band hole. The trion $\left(X^{-}\right.$, Bohr radius $\left.a_{\mathrm{tr}}\right)$ is bound (unbound) at low (high) electron density.

with photoluminescence (PL) and absorption spectroscopy. In the PL experiment, a laser with photon energy $1.959 \mathrm{eV}$ is just slightly blue-detuned with respect to the optical transition. Right-handed (left-handed) circularly polarized light $\sigma^{+}\left(\sigma^{-}\right)$injects a spin- $\downarrow$ (spin- $\left.\uparrow\right)$ hole at the $K$ point $\left(K^{\prime}\right.$ point) [Fig. 1(a)]. This spin-valley selectivity is crucial: the optical probe represents a spin-sensitive probe of the electronic ground state. Furthermore, PL represents a local measurement: information is gleaned from a few-hundred nanometer diameter region on the sample. On this length scale, inhomogeneous broadening in the optical response is small $[20,21]$.

$\mathrm{PL}$ in the presence of a Fermi sea of electrons has been explored in a number of 2D semiconductor systems ( $\mathrm{GaAs}$ [4,5], CdTe [22], $\left.\mathrm{MoSe}_{2}[23,24]\right)$ and there is a model to describe it $[25,26]$. At very low $n, \mathrm{PL}$ arises from the recombination of tightly bound electron-hole pairs, excitons $\left(X^{0}\right)$. As $n$ increases, $X^{0}$ weakens and is replaced with a redshifted peak, the trion $\left(X^{-}\right)$. The trion consists of two electrons in a spin-singlet state and a hole, or, in more accurate language, the bound exciton-Fermi sea polaron $[27,28]$. At higher $n$, the trions become unbound. In a simple picture, this occurs once an electron from the Fermi sea resides "inside" the trion wave function [Fig. 1(c)]. In PL, the $X^{-}$peak evolves gradually into a broad peak, typically redshifted with respect to $X^{-}$, the Mahan exciton [29]. A key property of the Mahan exction is the redshift of $\mathrm{PL}$ with respect to absorption, $E_{\mathrm{PL}}-E_{A}<0[25,26]$. In the single-particle limit for equal electron and hole masses (as for TMDCs), this shift is twice the Fermi energy.

We turn to gated $\mathrm{MoS}_{2}$. The absorption spectra at large out-of-plane magnetic field (two trions in $\sigma^{+}$polarization but no trions in $\sigma^{-}$polarization) show that the spins are polarized (ferromagnetism) at low-to-intermediate densities [6]. We focus here on PL. At $n \simeq 0$, the PL spectrum consists of a single sharp line corresponding to $X^{0}$ emission (Fig. 2) [30]. On increasing $n, X^{0}$ weakens and is replaced with a redshifted line, $X^{-}$(Fig. 2) $[6,23,24]$. At higher densities, $X^{-}$disappears and a broad peak (labeled $Q$ in Fig. 2) appears to the red. The $Q$ peak exhibits the large PL-absorption splitting characteristic of the Mahan exciton. These features follow the standard behavior of PL in the presence of a Fermi sea. There is however a radically different feature. Strikingly, the smooth transition between trion $\left(X^{-}\right)$and Mahan exciton $(Q)$ is missing: instead, there is an abrupt change from one to the other resulting in a "gap" in the PL spectrum (Fig. 2). This signals an abrupt change in the Fermi sea: it is the first evidence for a firstorder phase transition. The second evidence comes from the polarization of the PL. We excite and detect in all four combinations of circular polarization, presenting the results as a matrix (Fig. 2). For the $Q$ peak, the response is overwhelmingly "diagonal," i.e., polarization preserving: excitation with $\sigma^{+}\left(\sigma^{-}\right)$results in $\sigma^{+}$-polarized $\left(\sigma^{-}\right.$polarized) PL; Conversely, for $X^{-}$, there is a large "nondiagonal" response $\left(\sigma^{-}\right.$-polarized excitation results in $\sigma^{+}$-polarized PL yet $\sigma^{+}$-polarized excitation results in a very weak PL signal), clear evidence for symmetry breaking. The switch from one symmetry class to the other is also abrupt as the density changes.

We plot $E_{\mathrm{PL}}-E_{A}$ as a function of $n$ [Fig. 3(a)]. There is an abrupt change at $3.0 \times 10^{12} \mathrm{~cm}^{-2}$ signifying an abrupt change in the nature of the PL process. Also, we analyze the polarization dependence via

$$
\left(\begin{array}{l}
P_{+} \\
P_{-}
\end{array}\right)=\left(\begin{array}{cc}
a_{1}+h & a_{2} \\
a_{3} & a_{1}-h
\end{array}\right)\left(\begin{array}{l}
L_{+} \\
L_{-}
\end{array}\right)
$$

where $L_{+}\left(L_{-}\right)$is the $\sigma^{+}$-polarized $\left(\sigma^{-}\right.$-polarized) laser intensity, $P_{+}\left(P_{-}\right)$the $\sigma^{+}$-polarized $\left(\sigma^{-}\right.$-polarized $) \mathrm{PL}$ signal. $P_{+}$and $P_{-}$are integrated over the spectral window (Fig. 2). The term $a_{1}$ describes the polarization-preserving response. Terms $a_{2}$ and $a_{3}$ describe a transfer of the polarization, from $\sigma^{-}$at the input to $\sigma^{+}$at the output $\left(a_{2}\right)$; from $\sigma^{+}$at the input to $\sigma^{-}$at the output $\left(a_{3}\right)$. There is one further term. In the experiment, a hole is injected either at the $K$ point or at the $K^{\prime}$ point by choosing the polarization [Fig. 1(a)]. This works well but imperfectly. In an applied magnetic field, the hole can relax from the $K$ point to the $K^{\prime}$ point, a process is described by term $h$. We define the "PL polarization transfer" as $a_{2} /\left(a_{1}+a_{2}+a_{3}\right)$, i.e., the fraction of the total PL emitted in the polarization-nonconserving channel. As a function of $n$, the PL polarization transfer reaches values as high as $50 \%$ at intermediate density, decreasing rapidly between $n=2.5 \times 10^{12}$ and $n=3.5 \times$ $10^{12} \mathrm{~cm}^{-2}$ [Fig. 3(b)]. The opposite polarization-nonconserving process, $a_{3} /\left(a_{1}+a_{2}+a_{3}\right)$, is small at all $n$ (Fig. 3(b)). Both $E_{\mathrm{PL}}-E_{A}$ and the PL polarization transfer change abruptly at the same electron density. We identify a critical density of $n_{c}=3.0 \times 10^{12} \mathrm{~cm}^{-2}$ (Fig. 3).

Without a magnetic field, the abrupt change in $E_{\mathrm{PL}}-E_{A}$ is still clearly visible showing that there is still a phase transition at $n=n_{c}$ between states with different magnetic 


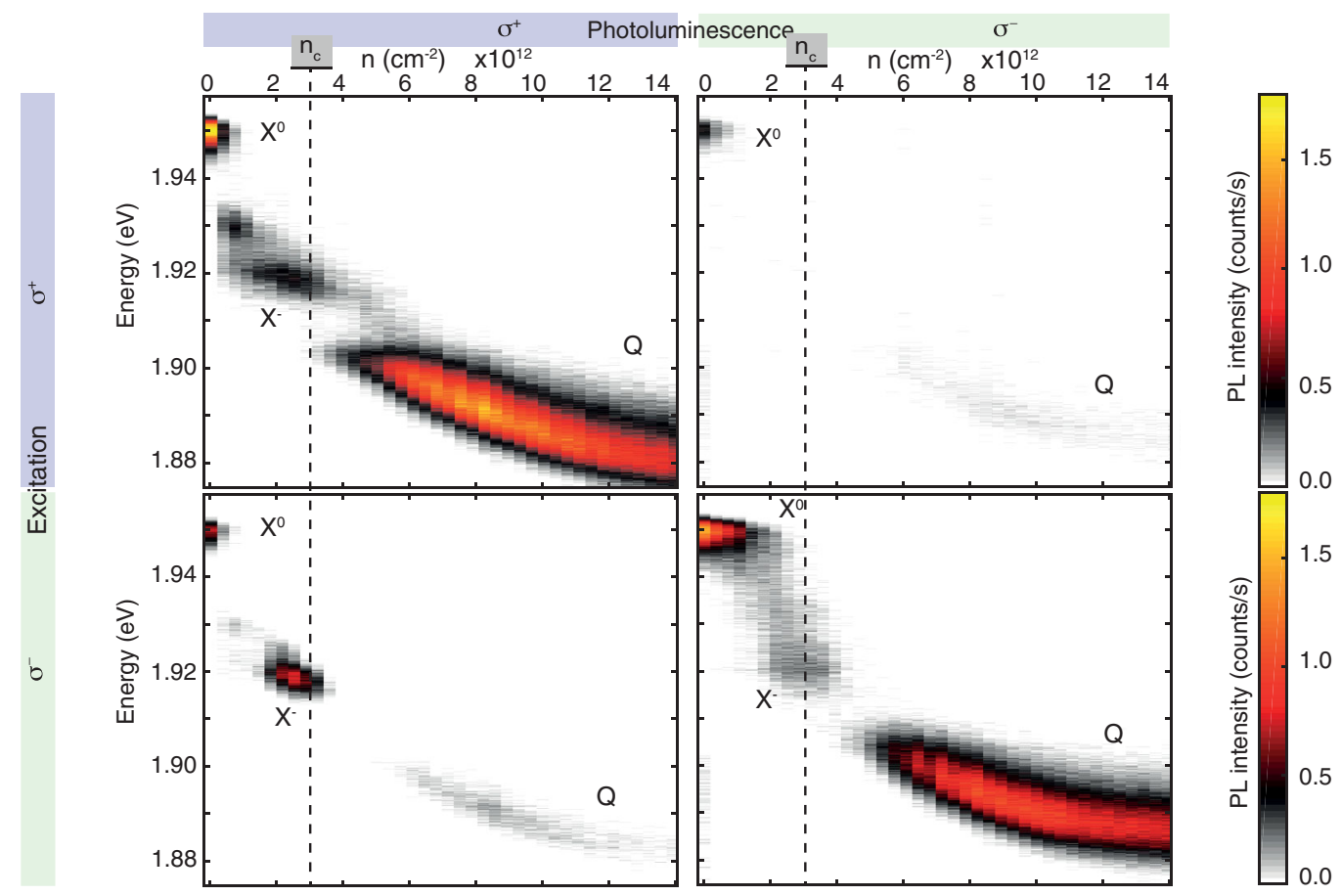

FIG. 2. Photoluminescence of gated monolayer-MoS 2 at $1.6 \mathrm{~K}$ and $B_{z}=9.00 \mathrm{~T}$. The excitation is either $\sigma^{+}$or $\sigma^{-}$; the detection either $\sigma^{+}$or $\sigma^{-}$: the top (bottom) row corresponds to $\sigma^{+}\left(\sigma^{-}\right)$excitation; the left (right) column to $\sigma^{+}\left(\sigma^{-}\right)$detection. $n_{c}$ denotes the critical density. At $n=n_{c}$ there is a jump in the PL energy: the PL process changes from a trion $\left(X^{-}\right)$to a Mahan exciton (Q). For $n>n_{c}$, the PL process is largely polarization conserving. Conversely, for $n<n_{c}$, the PL is highly polarization nonconserving: $\sigma^{-}$excitation leads to strong $\sigma^{+}$PL.

order [31]. However, there is zero net magnetization at $B_{z}=0$ (zero polarization transfer). At $B_{z}=0$, we propose that for $n<n_{c}$ there are fluctuating "puddles" of spin- $\uparrow$ and spin- $\downarrow$ electrons such that the net magnetization, averaged over time and space, goes to zero. This is consistent with the Ising symmetry of the system at low temperature: the spin-orbit interaction in the conduction band forces the magnetization to lie either in the "up" or "down" direction but in the absence of an applied magnetic field, both directions are equally likely. The size of the "puddles" is much smaller than the extent of the spatial probe in this experiment. At $B_{z}=0$ and $n>n_{c}$, the puddles disappear-there is no magnetic ordering whatsoever. This line of thinking explains the role of the magnetic field in these experiments-it establishes a quantization axis, favoring either the up or the down direction, and it allows long-range magnetic order stabilizing the ferromagnetic state. (We note that the Zeeman splitting even at $B_{z}=+9.00 \mathrm{~T}$ is an order of magnitude smaller than the Fermi energy in these experiments precluding magnetic order via a paramagnetic response. Also, if the response were solely paramagnetic then a phase transition would not occur, ruling out a giant paramagnetism.) In a large $B_{z}$ but at $T=30 \mathrm{~K}$, there is evidence that the phase transition survives [31].

Our results are in agreement with recent theory [37], which predicted spin ordering (and not ordering in the valley index or combined spin-valley index [38,39]) and a first-order phase transition between ferromagnetic and paramagnetic phases. Both predictions in the theory depend on corrections to Fermi liquid theory which arise via infrared electron-hole excitations at the Fermi energy [12-14]. This theory allows us to present a model that is fully consistent with the experimental results (Fig. 4). For $n<n_{c}$, the two bands with spin- $\uparrow$ are occupied resulting in a ferromagnetic state with a large spin polarization [6]; for $n>n_{c}$, all four bands are occupied, the ferromagnetism is destroyed and the spin polarization disappears [Fig. 4(a)]. The free energy $F$ depends on the magnetization fraction $M$ according to

$$
F=a M^{2}+b M^{4}+c|M|^{3} .
$$

The first two terms represent the Ginzburg-Landau model which, alone, lead to a second-order phase transition. The third term is a nonanalytic correction. Crucially, theory predicts a negative $c$ for $\mathrm{MoS}_{2}$ [37]. Plots of $F$ as a function of density (i.e., versus $a$ ) show how a negative $c$ leads to a first-order transition between paramagnetic and ferromagnetic phases [Fig. 4(b)]. We stress that the first-order nature of the phase transition depends on the nonanalytic correction: without it, the transition would be second order.

In the ferromagnetic (FM) phase, we observe PL from the trion: the trion is bound; in the paramagnetic (PM) 

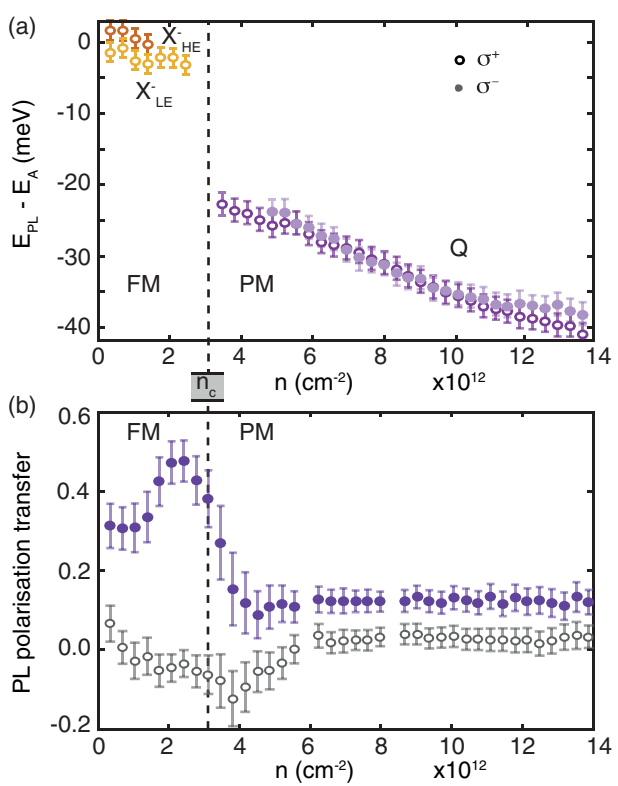

FIG. 3. (a) The energetic separation between the PL and the absorption, $E_{\mathrm{PL}}-E_{A}$ (measured either with $\sigma^{+}$or $\sigma^{-}$polarization), versus electron density at $1.6 \mathrm{~K}$ and $B_{z}=9.00 \mathrm{~T}$. There are two trions, $\mathrm{X}_{\mathrm{LE}}^{-}$and $\mathrm{X}_{\mathrm{HE}}^{-}$(red and orange points, respectively) [6]. The $Q$ peak is plotted for both polarizations $\left(\sigma^{+}\right.$and $\sigma^{-}$, openpurple and filled-purple points, respectively). (b) The PL polarization transfer $\left[a_{2} /\left(a_{1}+a_{2}+a_{3}\right)\right.$ (purple) and $a_{3} /\left(a_{1}+a_{2}+a_{3}\right)$ (white), see Eq. (1)], versus electron density. These plots determine $n_{c}=3.0 \times 10^{12} \mathrm{~cm}^{-2}$.

phase we do not observe PL from the trion: the trion is unbound. In other words, there is an abrupt change in the nature of the PL at $n_{c}$, from trion to Mahan exciton. This implies a profound change of the electron mass on passing from the FM to the PM phase. We analyze the simple concept [Fig. 1(c)]. The Fermi sea reduces the trion binding energy $E_{T}$ because all states with $k<k_{F}$ are occupied and become unavailable in constructing the trion wave function: $E_{T}=E_{T}^{0}-\hbar^{2} k_{F}^{2} / 2 \mu$. $\left(k_{F}\right.$ is the Fermi wave vector, $E_{T}^{0}$ the trion binding energy in the limit $n \rightarrow 0$, and $\mu$ the reduced mass of an exciton and an electron. This result comes from the Suris model [27] with the approximation $E_{T}^{0} \gg \hbar^{2} k_{F}^{2} / 2 \mu$.) In the FM (PM) phase, two (four) bands are occupied such that $E_{T}=0$ at $n_{\mathrm{FM}}=\mu E_{T}^{0} / \pi \hbar^{2}$ $\left(n_{\mathrm{PM}}=2 \mu E_{T}^{0} / \pi \hbar^{2}\right)$. The experiment tells us that $n_{\mathrm{FM}}$ must be larger than $n_{c}$, and that $n_{\mathrm{PM}}$ must be smaller than $n_{c}$, i.e., $n_{\mathrm{FM}}>n_{\mathrm{PM}}$. This conundrum can only be resolved by a change in the reduced mass, $\mu_{\mathrm{FM}}>2 \mu_{\mathrm{PM}}$, i.e., a significant decrease in the electron mass on going from the FM to the PM phase. Taking $E_{T}^{0}=17 \mathrm{meV} \mathrm{[6]} \mathrm{and} \mathrm{a} \mathrm{hole}$ mass of $m_{h}=0.54 m_{o}$ [40], we find $m_{e}^{\mathrm{FM}}>0.65 m_{o}$ and $m_{e}^{\mathrm{PM}}<0.40 m_{o}$. The mass in the ferromagnetic phase $\left(m_{e}^{\mathrm{FM}}\right)$ is consistent with the value deduced from Shubnikov-de Haas oscillations at low density $\left(0.8 m_{o}\right)$ [19]; the mass in the paramagnetic phase $\left(m_{e}^{\mathrm{PM}}\right)$ is consistent with the calculated bare electron mass $\left(0.44 m_{o}\right)$ [17].

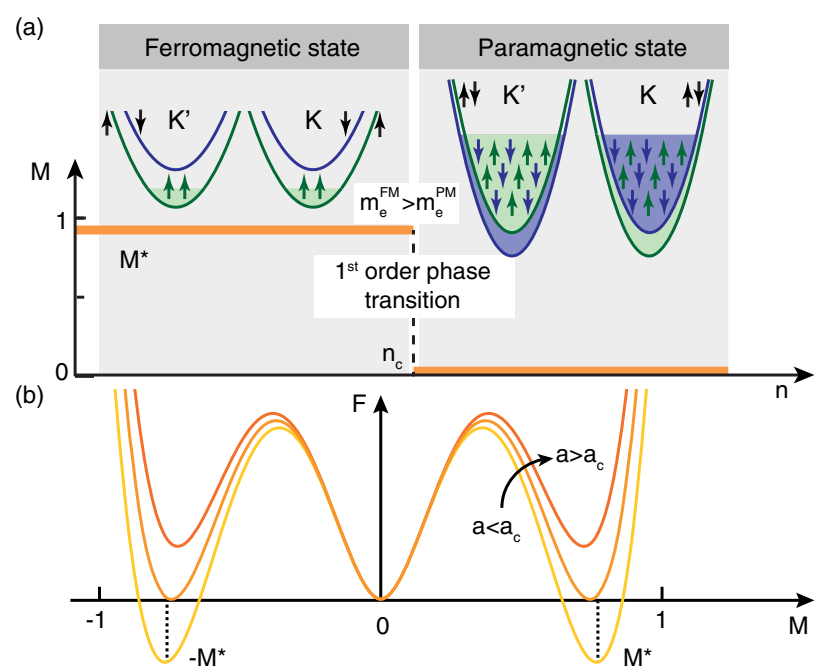

FIG. 4. (a) The occupied bands in the ferromagnetic phase (left) and in the paramagnetic phase (right) along with the change in magnetization at $n=n_{c}$. In the ferromagnetic phase, occupation of two bands with the same spin is favored, a consequence of exchange. In the paramagnetic phase, all four bands are occupied. (b) The free energy $F$ as a function of magnetization fraction $M$ for $F=a M^{2}+b M^{4}+c|M|^{3}$. The terms $a M^{2}$ and $b M^{4}$ are the Ginzburg-Landau terms; the nonanalytic term $c|M|^{3}$ arises from corrections to Landau's theory of the Fermi liquid. Plotted is $F$ for constant $b$ and $c$ with $c<0$ as a function of $a$. For $a<c^{2} / 4 b$ $\left(a>c^{2} / 4 b\right)$, the global minimum of $F$ lies at $M=0$, a paramagnetic phase (large $M$, a ferromagnetic phase). The phase transition at $a=c^{2} / 4 b$ is first order.

This analysis is consistent with general expectations that the mass is enhanced above its bare value in an interacting phase.

As an outlook, we comment that, first, our work establishes $\mathrm{MoS}_{2}$ as a platform for studying and exploiting interaction-driven physics. The combination of an ultrasmall Bohr radius, spin and valley degrees of freedom, and a small spin-orbit interaction creates a rich test bed. Second, the ferromagnetic-to-paramagnetic phase transition can be directly probed with a sensitive magnetometer. Finally, the first-order phase transition between ferromagnetic and paramagnetic phases enables the spin ordering to be controlled simply via a small change to a gate voltage opening a route to fast and efficient electrical switching. The next step is to stabilize the ferromagnetic phase without a large external field, for instance, via coupling to an insulating 2D ferromagnet.

We thank Mansour Shayegan for fruitful discussions. The work was supported by SNF (Project No. 200020_156637), the Georg H. Endress foundation, QCQT, SNI (Swiss Nanoscience Institute) and NCCR QSIT. K. W. and T. T. acknowledge support from the Elemental Strategy Initiative conducted by the MEXT, Japan and the CREST(JPMJCR15F3), JST. 
*jonasgael.roch@unibas.ch

[1] F. Bloch, Z. Phys. 57, 545 (1929).

[2] J. Zhu, H. L. Stormer, L. N. Pfeiffer, K. W. Baldwin, and K. W. West, Phys. Rev. Lett. 90, 056805 (2003).

[3] K. Vakili, Y. P. Shkolnikov, E. Tutuc, E. P. De Poortere, and M. Shayegan, Phys. Rev. Lett. 92, 226401 (2004).

[4] G. Finkelstein, H. Shtrikman, and I. Bar-Joseph, Phys. Rev. Lett. 74, 976 (1995).

[5] G. Yusa, H. Shtrikman, and I. Bar-Joseph, Phys. Rev. B 62, 15390 (2000).

[6] J. G. Roch, G. Froehlicher, N. Leisgang, P. Makk, K. Watanabe, T. Taniguchi, and R. J. Warburton, Nat. Nanotechnol. 14, 432 (2019).

[7] A. L. Sharpe, E. J. Fox, A. W. Barnard, J. Finney, K. Watanabe, T. Taniguchi, M. A. Kastner, and D. GoldhaberGordon, Science 365, 605 (2019).

[8] M. Brando, D. Belitz, F. M. Grosche, and T. R. Kirkpatrick, Rev. Mod. Phys. 88, 025006 (2016).

[9] E. C. Stoner, Proc. R. Soc. A 165, 372 (1938).

[10] N. D. Mermin and H. Wagner, Phys. Rev. Lett. 17, 1133 (1966).

[11] D. Loss, F. L. Pedrocchi, and A. J. Leggett, Phys. Rev. Lett. 107, 107201 (2011).

[12] D. Belitz, T. R. Kirkpatrick, and T. Vojta, Phys. Rev. Lett. 82, 4707 (1999).

[13] A. V. Chubukov, C. Pépin, and J. Rech, Phys. Rev. Lett. 92, 147003 (2004).

[14] D. L. Maslov and A. V. Chubukov, Phys. Rev. B 79, 075112 (2009).

[15] K. F. Mak, C. Lee, J. Hone, J. Shan, and T. F. Heinz, Phys. Rev. Lett. 105, 136805 (2010).

[16] D. Xiao, G.-B. Liu, W. Feng, X. Xu, and W. Yao, Phys. Rev. Lett. 108, 196902 (2012).

[17] A. Kormányos, V. Zolyomi, N. D. Drummond, and G. Burkard, Phys. Rev. X 4, 011034 (2014).

[18] K. Marinov, A. Avsar, K. Watanabe, T. Taniguchi, and A. Kis, Nat. Commun. 8, 1938 (2017).

[19] R. Pisoni, A. Kormányos, M. Brooks, Z. Lei, P. Back, M. Eich, H. Overweg, Y. Lee, P. Rickhaus, K. Watanabe, T. Taniguchi, A. Imamoglu, G. Burkard, T. Ihn, and K. Ensslin, Phys. Rev. Lett. 121, 247701 (2018).

[20] F. Cadiz, E. Courtade, C. Robert, G. Wang, Y. Shen, H. Cai, T. Taniguchi, K. Watanabe, H. Carrere, D. Lagarde, M. Manca, T. Amand, P. Renucci, S. Tongay, X. Marie, and B. Urbaszek, Phys. Rev. X 7, 021026 (2017).

[21] O. A. Ajayi, J. V. Ardelean, G. D. Shepard, J. Wang, A. Antony, T. Taniguchi, K. Watanabe, T. F. Heniz, S. Strauf, X.-Y. Zhu, and J. C. Hone, 2D Mater. 4, 031011 (2017).
[22] V. Huard, R. T. Cox, K. Saminadayar, A. Arnoult, and S. Tatarenko, Phys. Rev. Lett. 84, 187 (2000).

[23] M. Sidler, P. Back, O. Cotlet, A. Srivastava, T. Fink, T. Korner, E. Demler, and A. Imamoglu, Nat. Phys. 13, 255 (2017).

[24] P. Back, M. Sidler, O. Cotlet, A. Srivastava, N. Takemura, M. Kroner, and A. Imamoğlu, Phys. Rev. Lett. 118, 237404 (2017).

[25] T. Uenoyama and L. J. Sham, Phys. Rev. Lett. 65, 1048 (1990).

[26] P. Hawrylak, Phys. Rev. B 44, 3821 (1991).

[27] R. A. Suris, V. Kochereshko, G. Astakhov, D. Yakovlev, W. Ossau, J. Nürnberger, W. Faschinger, G. Landwehr, T. Wojtowicz, G. Karczewski, and J. Kossut, Phys. Status Solidi B 227, 343 (2001).

[28] D. K. Efimkin and A. H. MacDonald, Phys. Rev. B 95, 035417 (2017).

[29] G. D. Mahan, Phys. Rev. 153, 882 (1967).

[30] A. Chernikov, T. C. Berkelbach, H. M. Hill, A. Rigosi, Y. Li, O. B. Aslan, D. R. Reichman, M. S. Hybertsen, and T. F. Heinz, Phys. Rev. Lett. 113, 076802 (2014).

[31] See Supplemental Material at http://link.aps.org/ supplemental/10.1103/PhysRevLett.124.187602 for details on the sample fabrication and on the experimental setup. Additional data are also presented and discussed in the Supplemental Material. The Supplemental Material contains Refs. [6,19,32-36].

[32] P. Zomer, M. Guimarães, J. Brant, N. Tombros, and B. Van Wees, Appl. Phys. Lett. 105, 013101 (2014).

[33] T. Taniguchi and K. Watanabe, J. Cryst. Growth 303, 525 (2007).

[34] L. Ju, J. J. Velasco, E. Huang, S. Kahn, C. Nosiglia, H.-Z. Tsai, W. Yang, T. Taniguchi, K. Watanabe, and Y. Zhang, Nat. Nanotechnol. 9, 348 (2014).

[35] A. Epping, L. Banszerus, H. Guttinger, L. Kruckeberg, K. Watanabe, T. Taniguchi, F. Hassler, B. Beschoten, and C. Stampfer, J. Phys. Condens. Matter 30, 205001 (2018).

[36] H. Wang, Y. Wu, C. Cong, J. Shang, and T. Yu, ACS Nano 4, 7221 (2010).

[37] D. Miserev, J. Klinovaja, and D. Loss, Phys. Rev. B 100, 014428 (2019).

[38] M. Van der Donck and F. M. Peeters, Phys. Rev. B 98, 115432 (2018).

[39] J. E. H. Braz, B. Amorim, and E. V. Castro, Phys. Rev. B 98, 161406(R) (2018).

[40] A. Kormanyos, G. Burkard, M. Gmitra, J. Fabian, V. Zolyomi, N. D. Drummond, and V. Fal'ko, 2D Mater. 2, 022001 (2015). 